\title{
Taking RAS research to space
}

\author{
Albert H. Chan ${ }^{a}$, Anna Maciag ${ }^{b}$, Dwight Nissleyc, and Dhirendra K. Simanshu ${ }^{d}$ \\ $\mathrm{NCI}$ RAS Initiative, Cancer Research Technology Program, Frederick National Laboratory for \\ Cancer Research, Frederick, MD 21702. ${ }^{a}$ albert.chan@nih.gov; ${ }^{b}$ anna.maciag2@nih.gov; ${ }^{c}$ \\ nissleyd@mail.nih.gov; ${ }^{d}$ dhirendra.simanshu@nih.gov
}

Oncogenic RAS mutations are responsible for close to $30 \%$ of all human cancers [1]. Despite decades of research, there are no effective cancer drugs that target RAS oncoproteins. KRAS protein contains a highly conserved GTPase-domain (G-domain) and diverges substantially in the last 20 amino acids at the C-terminal end known as the hypervariable region (HVR) [1]. HVR undergoes multiple post-translational modifications including farnesylation at Cys185, and this modified HVR is important for KRAS association with the membrane, which in turn is essential for activating downstream signaling [2]. Interestingly, our molecular dynamics simulations and NMR studies suggest direct interaction of the unmodified KRAS4b HVR with the G-domain, at least transiently. Small molecule inhibitors can be developed to stabilize the unmodified HVR:Gdomain interaction to prevent HVR modification and membrane association. We attempted to solve the crystal structure of the full length KRAS4b with the unmodified HVR. Unfortunately, the electron density of the HVR is not visible with the crystals grown in our laboratory. In order to improve data quality to capture the HVR density, we partnered with the Center for the Advancement of Science in Space (CASIS) to crystallize KRAS proteins on the International Space Station (ISS) under microgravity environment. We used commercially available hardware including 96-well plates, 72-well microbatch trays, and capillaries to perform crystallization. Crystallization samples were set up in the laboratory and immediately frozen prior to launching to the ISS in the SpX-16 mission. Samples were thawed on the ISS to allow crystallization to proceed in microgravity environment. Despite only using regular commercially available hardware, we achieved excellent samples recovery upon samples return, and many of the drops contained crystals. Compared to the crystals grown in our laboratory, overall the microgravity crystals have better appearances, significantly better mosaicity and much improved signal-to-noise.

\section{References}

[1] Hobbs, G.A., Der, C.J. \& Rossman, K.L. (2016) J Cell Sci, 129, 1287-1292

[2] Ostrem, J.M.L. \& Shokat, K.M. (2016) Nat Rev Drug Discov, 15, 771-785 